【研究简报】

\title{
分子构型控制合成芳香聚酯的预聚体: 芳香酯环状低聚物
}

\author{
姜洪炎 陈天禄 张越 何天白 徐纪平 \\ (中国科学院长春应用化学研究所, 长春 130022)
}

\section{关链词 分子构型 芳香酯环状低聚物 晶体结构 开环聚合}

芳香聚酯是一类重要的工程材料, 具有强度高、耐高温、耐溶剂等特点. 它们通常是用溶. 液聚合或熔融聚合的方法来制备的. 所得聚合物溶解性差, 熔融粘度高, 极大地限制了这些优 良材料的应用, 特别是在复合材料领域的应用. 开环聚合无疑是解决这一难题的有效途径之 - [1]. 然而, 环状低聚物合成产率低, 同时伴随着线性副产物生成的事实一直困扰着化学家 们. 在芳香酯环状低聚物的合成过程中, 我们发现反应物的分子构型对于环状低聚物的产率 有着较大的影响. 通过分子设计可以高产率地合成芳香酯环状低聚物, 而且在阴离子催化剂 存在条件下, 芳香酯环状低聚物熔融开环聚合可得到高分子量的线性芳香聚酯.

\section{1 实验}

（1）芳香酯环状低聚物的合成. 称取 $1.5227 \mathrm{~g}(7.5 \mathrm{mmol})$ 邻苯二甲酰氯, $1.7121 \mathrm{~g}$ (7.5 mmol) 双酚 $\mathrm{A}$, 分别配成 $50 \mathrm{~mL}$ 的二氯甲烷和氢氧化钠水溶液. 将两种反应溶液分别盛. 在两只自制可控制滴加速度的注射器中, 在 $8 \mathrm{~h}$ 内缓慢、等速地滴加到盛有 $200 \mathrm{~mL}$ 二策甲烷, $30 \mathrm{~mL}$ 水, $0.2 \mathrm{~g}$ 十六烷基三甲基溴化铵, 剧烈搅拌的 $500 \mathrm{~mL}$ 三口瓶中, 反应温度为室温. 反

\section{参考 文 献}

1 Diederich F, Thilgen C. Covalent fullerene chemistry. Science, 1996, 271(5247): 317 323

2 Hirsch A. The Chemistry of the Fullerenes. Stuttgart: Thieme, 1994. 79 114

3 Maggini M, Scorrano G, Prato M. Addition of azomethine yiides to $\mathrm{C}_{60}$ : synthesis, characterization, and functionlization of fullerene pyrrolidine. J Am Chem Soc, 1993, 115(21): $9798 \sim 9799$

4 Shu L, Wang G, Wu S, et al. 1,3-Dipolar cycloaddition reaction of [60] fullerene and imines of $\alpha$-amino acid ester: formation of fullerene-fused proline derivatives. Tetrahedron Lett, 1995, 36(22): $3871 \sim 3874$

5 Han H X, Li Y L, Li Y F, et al. Preparation and characterization of a new hexahydropyrrolizine derivative of fullerene. Chin Chem Lett, 1995, 6(3): $201 \sim 204$

6 Zhou D J, Gan L B, Luo C P, et al. Syntheis of fullerene pyrrolidines containing substituted phenyl group through azomethine ylide addition. Chin Chem Lett, 1995, 6(12): $1033 \sim 1036$

7 Zhou D J, Gan L B, Tan H S, et al. Langmuir-Blodgett films and photophysical properties of a $\mathrm{C}_{60}$-sarcosine methyl ester derivative, $\mathrm{C}_{60}\left(\mathrm{C}_{3} \mathrm{H}_{9} \mathrm{NO}_{2}\right)$. J Phys Chem, 1996, 100(8): $3150 \sim 3156$

8 Zhou D J, Tan H S, Luo C P, et al. Fullerene induced C- N bond breaking and formation: synthesis of fullerene pyrrolidine and methanofullerene sarcosine derivatives by photochemical addition of sarcosine ester to $\mathrm{C}_{60}$. Tetrahedron Lett, 1995,36 (50): $9169 \sim 9172$ 
应结束后, 继续摚拌 $30 \mathrm{~min}$. 分液得到有机相, 用蒸馏水洗至中性, 蒸干有机相得到 $2.52 \mathrm{~g}$ 白 色固体, 产率 $94 \%$. 四溴双酚 $\mathrm{A}$ 芳香酯用同样的方法合成, 环状二聚体的产率为 $63 \%$.

(2) 四澳双酚 A 芳香酯环状二聚体 (BPA-Br) 的晶体结构分析. 将在二氯甲烷中培养出 来的无色晶体从溶液中取出, 马上用硅胶包裹, 在 Siemens P4 四圆衍射仪上用单色化的 MoK $\alpha$ $(\lambda=0.071073 \mathrm{~nm})$ 射线, 以 $\omega / 2 \theta$ 扫描方式, 在 $1.62^{\circ} \leqslant \theta \leqslant 25.01^{\circ}$ 范围内收集到 9561 个独立 的衍射数据, 用其中 $I>2 \sigma(I)$ 的数据参加结构修正. 此环状化合物属于三斜晶系, 空间群 P1. 晶胞系数 $a=1.3964(2), b=1.4341$ (2), $c=1.7004(8) \mathrm{nm} ; \alpha=87.830$ (10), $\beta=$ $70.170(10), \gamma=62.320(10)^{\circ} ; V=2.8072(14) \mathrm{nm}^{3}, Z=2, M_{\mathrm{r}}=1526.03, D_{\mathrm{x}}=1.805$ $\mathrm{g} / \mathrm{cm}^{3}, \mu=5.863 \mathrm{~mm}^{-1}, \mathrm{~F}(000)=1486$. 直接用 SHELXTL PLUS 程序解析结构, 对非氢原 子用各向异性热参数进行全矩阵最小二乘法修正, 氢原子采用差值 Fourier 合成法找到. 最后 偏离因子 $R=0.0986$. 最后一轮差值 Fourier 图上的残余电子密度为 $1.200 \mathrm{e} /(0.1 \mathrm{~nm})^{3}$.

\section{2 结果与讨论}

最近, 我们发现在“拟高稀释的模拟” (Pseudo-high-dilution) ${ }^{[2]}$ 条件下, 即将邻苯二甲酰氯 和双酚 $\mathrm{A}$ 分别配成等摩尔浓度的二氯甲烷溶液和氢氧化钠水溶液, 在一定的时间内缓慢、匀 速地滴加到盛有相转移催化剂 (十六烷基三甲基溴化铵)剧烈摚拌的三口瓶中, 界面缩聚反应 生成了单一组分的产物 (GPC 分析呈现 1 个很窄的单一峰). 经波谱 (IR, MS, ${ }^{1} \mathrm{H} N \mathrm{NMR}$, ${ }^{13} \mathrm{C} \mathrm{NMR}$ ), 最后经单晶 $\mathrm{X}$-射线分析表明, 此产物为芳香酯环状二聚体. 分子模拟 ${ }^{[3]}$ 以及晶体 结构分析表明, 双酚 A 芳香酯环状二聚体 (BPA) 是一个没有张力或张力很小的环状化合物. 根据 Jacobson-Stockmayer 理论 ${ }^{[4]}$, 创造“拟高稀释的模拟”条件将有利于形成小环化合物. 因 此满足有利于环化构型的邻苯二甲酰氯和双酚 $\mathrm{A}$ 优先形成了没有张力的环状二聚体.

当以四溴双酚 $\mathrm{A}$ 代替双酚 $\mathrm{A}$, 在相同的反应条件下和邻苯二甲酰氯界面缩聚反应, 经

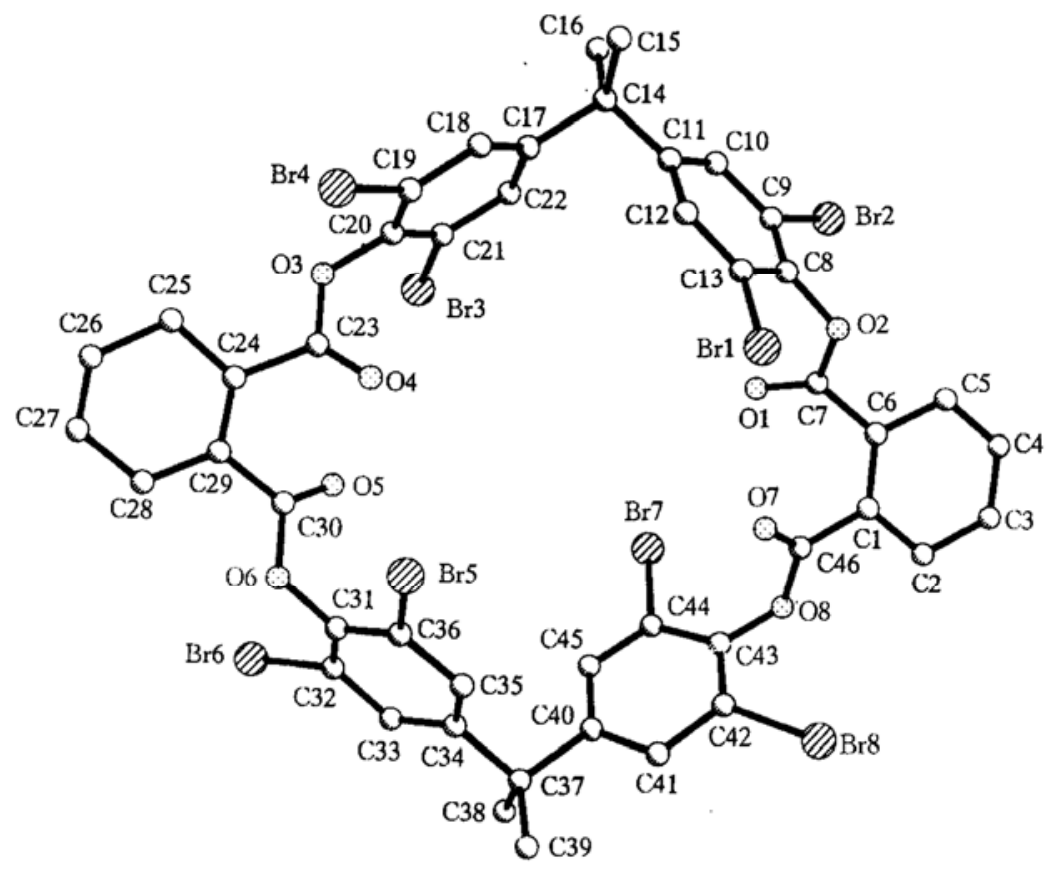

图 1 四溴双酚 $\mathrm{A}$ 芳香酯环状二聚体分子结构图
GPC 分析表明反应产物是由 $63 \%$ 的环状二聚体和 $37 \%$ 的大 环化合物 $(n \geqslant 3)$ 或聚合物组 成. 经过分离, 重结晶得到纯 的四溴双酚 $\mathrm{A}$ 芳香酯环状二聚 体(BPA-Br). 此环状二聚体的 单晶 X 射线分子结构如图 1 所 示. 非氢原子坐标和热参数见 表 1 .

我们发现 BPA-Br 的分子 构型与 BPA 的分子构型截然 不同. 最明显的是在 BPA-Br 中, 四个羰基都伸向环空腔的 内部, 而在 BPA 中, 有两个羰 基伸向环空腔的内部, 而另外 两个羰基伸向环空腔的外部. 大部分键角也被压小．例如键 
表 1 非氢原子坐标 $\left(\times 10^{4}\right)$ 和热参数 $\left[\left(10^{-2} \mathrm{~nm}^{2}\right) \times 10^{3}\right]$

\begin{tabular}{|c|c|c|c|c|c|c|c|c|c|}
\hline & $x$ & $y$ & $z$ & $U / \mathrm{eq}$ & & $x$ & $y$ & $z$ & $U /$ eq \\
\hline $\mathrm{Br}(1)$ & $-2956(2)$ & $10451(2)$ & 3315 (1) & $84(1)$ & $C(22)$ & $1268(14)$ & $6783(12)$ & $1060(11)$ & $39(5)$ \\
\hline $\mathrm{Br}(2)$ & $-1731(2)$ & $10802(2)$ & $-165(1)$ & $78(1)$ & C (23) & $2860(18)$ & 5857 (15) & $3060(13)$ & $51(5)$ \\
\hline $\mathrm{Br}(3)$ & 3407 (2) & $6643(2)$ & $921(2)$ & $82(1)$ & C (25) & 4256 (19) & $4103(15)$ & 3308 (13) & $71(7)$ \\
\hline $\mathrm{Br}(4)$ & $1105(2)$ & $4793(2)$ & 3268 (2) & $74(1)$ & C (25) & $4256(19)$ & $4103(15)$ & 3308 (13) & $71(7)$ \\
\hline $\mathrm{Br}(5)$ & $1302(2)$ & $8526(2)$ & $5090(2)$ & $76(1)$ & C (26) & $5166(20)$ & $3542(17)$ & $3645(13)$ & $80(8)$ \\
\hline $\mathrm{Br}(6)$ & $5865(2)$ & $7873(2)$ & $3714(2)$ & $99(1)$ & C (27) & $5633(23)$ & 3978 (17) & 3956 (14) & $81(8)$ \\
\hline $\mathrm{Br}(7)$ & $1622(2)$ & $11786(2)$ & $1660(1)$ & $88(1)$ & C & 5257 (17) & 5048 (15) & 3947 (11) & $57(6)$ \\
\hline $\mathrm{Br}(8)$ & $-2037(2)$ & $14637(2)$ & $4524(2)$ & $77(1)$ & C (29) & $4360(15)$ & $5683(14)$ & 3662 (11) & $46(5)$ \\
\hline$O(1)$ & $-887(12)$ & $11338(9)$ & $1432(9)$ & $63(4)$ & $C(30)$ & $4046(15)$ & $6825(13)$ & $3582(14)$ & $45(5)$ \\
\hline$O(2)$ & $-2668(10)$ & $11531(9)$ & $1732(8)$ & $50(3)$ & C & $3522(18)$ & 8408 (15) & 4374 & $46(5)$ \\
\hline$O(3)$ & 3033 (11) & $5345(9)$ & $2309(8)$ & $54(3)$ & C & 4226 (15) & $8798(16)$ & 4154 & \\
\hline $\mathrm{O}$ & 2050 & 66 & 341 & 5) & & 38 & 9916 & 42 & $68(6)$ \\
\hline O ( & 3920 & 7262 & 300 & $84(5)$ & & $2639(16)$ & 10616 & 45 & $45(5)$ \\
\hline$O(6)$ & $3896(10)$ & $7316(9)$ & 4338 (7) & $51(3)$ & & $1886(17)$ & 10194 & 4798 & $48(5)$ \\
\hline$O(7)$ & $-1466(13)$ & $12388(10)$ & $3029(9)$ & 67 & & 230 & 9065 & 4726 & $60(6)$ \\
\hline$O(8)$ & $-783(11)$ & $13584(10)$ & $2696(8)$ & 54 & C ( & $6(17)$ & 118 & 46 & 5) \\
\hline C (1) & $-2155(15)$ & $13673(16)$ & $2120(12)$ & $49(5)$ & D & 17) & 121 & 42 & \\
\hline$C(2)$ & $-2560(16)$ & $14760(15)$ & 2114 (12) & & & 19) & 122 & 5 & \\
\hline & -3313 & 152 & 16 & 65 & & 8) & 122 & 41 & $50(5)$ \\
\hline C (4) & $-3594(19)$ & 14709 (17) & (15) & $75(7)$ & C & 176 & 13096 & 45 & $50(5)$ \\
\hline$C(5)$ & $-3234(16)$ & 13609 (15) & 1305 (13) & $61(6)$ & C & $-498(15)$ & $13466(13)$ & 3962 (15) & $59(6)$ \\
\hline$C(6)$ & $-2442(14)$ & 13079 (14) & $1725(11)$ & $43(5)$ & C (43) & $-96(17)$ & 13079 (15) & 3152 (11) & 49 \\
\hline$C(7)$ & -1909 (17) & 11891 (15) & $1627(10)$ & $39(5)$ & C (44) & $1021(18)$ & 12293 (15) & 12) & \\
\hline$C(8)$ & $-2220(15)$ & $10456(14)$ & $1542(13)$ & $48(5)$ & C & 172 & 118 & 32 & \\
\hline & -1700 & 9972 (14) & $724(14)$ & 53 & C & 1460 & 13108 & 2679 (11) & $42(5)$ \\
\hline$C(10)$ & $-1160(17)$ & 8882 (17) & $516(14)$ & $63(6)$ & C (47) & $506(22)$ & 9869 (19) & $2653(19)$ & $109(10)$ \\
\hline C (11) & $-1155(16)$ & $8231(13)$ & $1138(12)$ & $42(5)$ & C (48) & $4938(39)$ & 9007 (31) & $1786(25)$ & $200(18)$ \\
\hline$C(12)$ & $-1772(16)$ & $8741(13)$ & $2002(13)$ & $49(5)$ & C (49) & $5142(30)$ & $9872(24)$ & $1845(22)$ & 282 \\
\hline & $-2200(18)$ & $9794(14)$ & $2172(13)$ & & $0)$ & $-6095(32)$ & $13282(30)$ & $629(24)$ & (16) \\
\hline C (14) & $-639(17)$ & $7034(14)$ & $996(11)$ & $47(5)$ & 1) & $-5374(30)$ & $12701(28)$ & 1048 (22) & $152(13)$ \\
\hline$C(15)$ & $-1514(15)$ & $6610(14)$ & $1360(12)$ & $56(5)$ & C1 (1) & $264(9)$ & $8845(8)$ & $2643(6)$ & $152(3)$ \\
\hline$C(16)$ & $-24(17)$ & $6593(13)$ & $45(12)$ & $62(6)$ & C1 (2) & $1790(10)$ & 9477 (9) & $2830(7)$ & $173(4)$ \\
\hline $\mathrm{C}(17)$ & $359(17)$ & $6572(13)$ & $1363(12)$ & $49(5)$ & $O(9)$ & $-5454(30)$ & 12149 (27) & $1692(21)$ & $243(14)$ \\
\hline$C(18)$ & 317 (17) & $5980(13)$ & $2056(11)$ & $45(5)$ & $O(10)$ & $-6948(22)$ & $13617(19)$ & $344(15)$ & $161(9)$ \\
\hline $\mathrm{C}(1$ & $1196(18)$ & $5580(12)$ & $2356(12)$ & $46(5)$ & $O(11)$ & $5080(30)$ & $8281(26)$ & $1094(21)$ & 245 \\
\hline & 2094 (17) & 5775 (13) & $2035(12)$ & $48(5)$ & OW & $5924(41)$ & $9323(37)$ & $408(30)$ & $35(21)$ \\
\hline $\mathrm{C}(21)$ & $2160(17)$ & $6384(13)$ & $1374(12)$ & $48(5)$ & & & & & \\
\hline
\end{tabular}

角 $\mathrm{C}-\mathrm{O}-\mathrm{C}$ 在 BPA 中为 118.6 (3) 和 112.0 (3) ${ }^{\circ}$ (理论值为 $118.8^{\circ[5]}$ ), 而在 BPA-Br 中分别 为 $117.0(14), 116.1(14), 114.7(13)$ 和 $111.4(3)^{\circ}$; 键角 $\mathrm{C}-\mathrm{C}(=\mathrm{O})-\mathrm{O}$ 在 BPA 中为 111.1 (3) 和 $110.5(3)^{\circ}$ (理论值为 $111.7^{\circ[5]}$ ), 而在 BPA-Br 中分别为 $114(2), 110(2), 109$ (2) 和 $109(2)^{\circ}$; 键角 phenyl-C-phenyl 在 BPA 中 108.6 (3) (理论值为 $109.3^{\circ}{ }^{[6]}$ ), 而在 BPA-Br 中分 别为 107 (2) 和 $106.2(14)^{\circ}$. 另外在 BPA-Br 中, 苯环也存在着不同程度的扭曲. 六个苯环的 碳原子偏离平面的均方根即 $\left(\Sigma z^{2} / 6\right)^{1 / 2}$ 分别为 $0.0004,0.0009,0.0010,0.0011,0.0013$ 和 $0.0028 \mathrm{~nm}$. 可见由于溴原子的引入改变了环状二聚体分子的构型, 给环状二聚体分子带来 了较大的张力, 结果环状二聚体优先生成的同时, 伴随着大环化合物 $(n \geqslant 3)$ 或聚合物的生成.

当以间苯二甲酰氯代替邻苯二甲酰氯在相同的反应条件下和双酚 $\mathrm{A}$ 界面缩聚反应, 结果 反应产物是由环状低聚物 (聚合度 $n=2 \sim 7$, 以环状三聚体为主) 和线性聚合物组成 ${ }^{[7]}$. 环状 低聚物总的产率为 $50 \%$. 计算机分子模拟间苯二甲酰氯和双酚 A 芳香酯环状二聚体结果表 明, 此环状二聚体分子是一个具有高度张力的环. 明显地, 键角 phenyl-C-phenyl 扩大到 


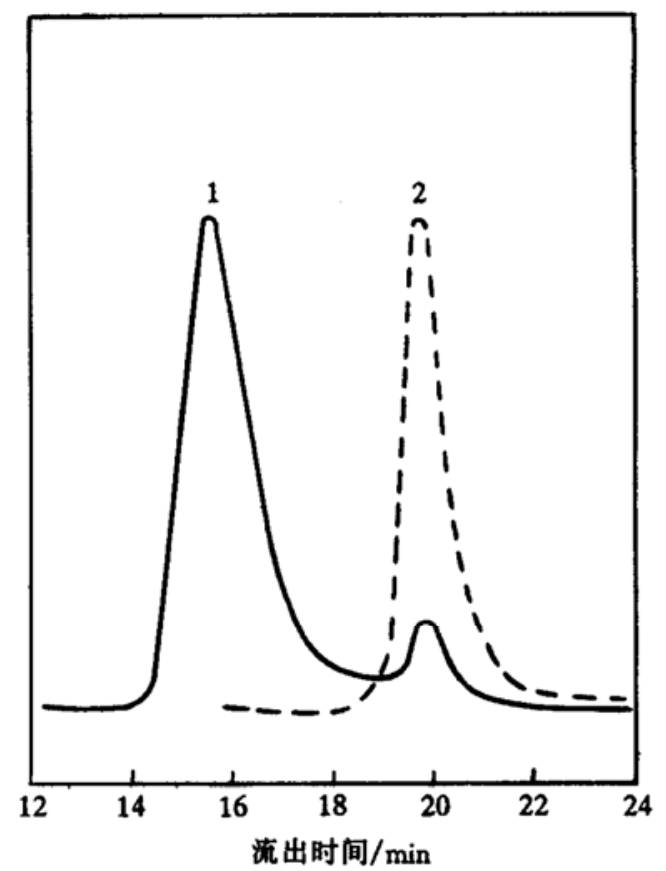

图 2 双酚 $\mathrm{A}$ 芳香酯环状二聚体及其 聚合产物的 GPC 曲线 1 为聚合产物, 2 为双䤅 $\mathrm{A}$ 芳香酯环状二聚体

$112.3^{\circ}$ 和 $113.1^{\circ}$, 键角 phenyl-C $(=\mathrm{O})-\mathrm{O}$ 分别为 $126.5^{\circ}, 123.1^{\circ}, 122.9^{\circ}$ 和 $120.4^{\circ}$ (理论值为 $111.7^{\circ}{ }^{[5]}$ ), 键角 $\left(\mathrm{O}=\mathrm{C}-\mathrm{O}-\right.$ phenyl 分别为 $123.3^{\circ}, 123.1^{\circ}$, $120.7^{\circ}, 114.1^{\circ}$ (理论值为 $118.8^{\circ[5]}$ ).

总之, 分子构型对芳香酯环状低聚物的产率影响 甚大, 了解分子构型和成环难易的关系, 对于环状化合 物的合成有着重要的指导意义.

芳香酯环状化合物可以通过酯交换反应 ${ }^{[8]}$ 开环聚 合得到高分子量的线性芳香聚酯. 双酚 $\mathrm{A}$ 芳香酯环状 化合物与 $0.1 \mathrm{~mol}$ 的四苯嗍四丁铵 (百分含量) 充分混 合, 在 $310^{\circ} \mathrm{C}$, 氮气保护条件下熔融聚合, $40 \mathrm{~min}$ 后可 拉出柔韧的丝. GPC 分析聚合产物, 发现少于 $5 \%$ 的 环状化合物留在聚合产物中, 然而链-链平衡发生很 快, 导致分子量分布约为 2.0. 双酚 A 芳香酯环状化 合物及其聚合产物的 GPC 曲线如图 2 所示.

聚合产物比浓粘度为 $0.48 \mathrm{dL} / \mathrm{g}(0.34 \%$ 的氯仿 溶液, $25^{\circ} \mathrm{C}$ ), 重均分子量为 96000 , 数均分子量为 51000 . 所得聚合物强度较大, 淡黄色透明, 溶解在许 多普通溶剂如二氯甲烷、氯仿和四氢呋喃中. 与对应 的环化物不同,所得聚合物呈无定型态. 玻璃化转变温度为 $148^{\circ} \mathrm{C}$ (DSC 测定, 升温速度为 $\left.10^{\circ} \mathrm{C} / \mathrm{min}, \mathrm{N}_{2}\right), 5 \%$ 失重温度为 $358^{\circ} \mathrm{C}$.

\section{参考文献}

1 姜洪烧天禄, 徐纪平, 等. 开环聚合扩展了热塑性树脂的加工和应用. 科学通报, 1996, 41(11):1055

2 Knops P, Sendhoff N, McKelburger H, et al. High dilution reaction-new synthetic applications. Top Curr Chem, 1991, $161: 1$

3 Tyuzyo K, Harada Y, Suzuki J. Cyclic condensation product from bisphenol A and $o$-phthaloyldichloride. Polym Lett, 1964, $2: 43$

4 Jacobson H, Stockmayer W H. Intramolecular reaction in polymerizations. J Chem Phys, 1950, 18:1 600

5 Coulter P, Windle A. Geometric and rotational parameters for conformational modeling of liquid crystalline polyesters. Macromolecules, 1989, 22:1 129

6 Stewart K R. Molecular simulation studies on the structure and conformation of cyclic polycarbonate oligomers. Polym Prepr (Am Chem Soc Div Polym Chem), 1989, 30(2):140

7 Guggenheim T L, McCormick S J, Kelly J J, et al: Synthesis and polymerization of cyclic oligomeric arylates based on bisphenol A and isophthaloyldichloride. Polym Prepr (Am Chem Soc Div Polym Chem), 1989, 30(2): 579

8 Brunelle D J. Cyclic carbonates. In: Brunelle D J ed. Ring-Opening Polymerization: Mechanism Catalysis Structure Utility. New York: Hanser, 1993. chap 11

(1996-06-21 收稿, 1997-01-29 收修改稿) 